\title{
Planar scintimammography and SPECT in neoadjuvant chemo or hormonotherapy response evaluation in locally advanced primary breast cancer
}

\author{
ANGELA SPANU $^{1}$, ANTONIO FARRIS ${ }^{2}$, FRANCESCA CHESSA ${ }^{1}$, DANIELA SANNA ${ }^{1}$, \\ MARIA PITTALIS $^{2}$, ALESSANDRA MANCA ${ }^{3}$ and GIUSEPPE MADEDDU ${ }^{1}$ \\ Departments of ${ }^{1}$ Nuclear Medicine, ${ }^{2}$ Oncology, and ${ }^{3}$ Pathology, \\ University of Sassari, Viale San Pietro 8, I-07100 Sassari, Italy
}

Received December 11, 2007; Accepted January 30, 2008

\begin{abstract}
Conventional imaging procedures have proved of limited value in assessing tumor response to neoadjuvant chemotherapy in locally advanced primary breast cancer (LAPBC). We evaluated the usefulness of radioisotopic procedures comparing planar scintimammography (SM) to SPECT, monitoring pre-surgery neoadjuvant chemo- or hormonotherapy response in $32 \mathrm{LAPBC}$ patients. In all cases, 99mTc-tetrofosmin conventional planar SM and SPECT were acquired by dual-head gamma camera with HR parallel hole collimators. In 15 cases, planar SM with small field of view high resolution dedicated breast camera (DBC) was also acquired. Scintigraphic data always correlated with histopathological findings. At surgery, 4/32 patients had pathological complete remission (pCR), while 28/32 patients had residual tumors. Both conventional planar SM and SPECT were true negative in $4 / 4(100 \%)$ pCR patients, as was DBC in the only studied case. Conventional planar SM and SPECT detected residual tumors in 23/28 (82\%) and in $25 / 28(89.2 \%)$ cases, respectively. Both procedures missed 2 multifocal, scattered microscopic residues, only evidenced at DBC. Conventional planar SM also missed 3 further macroscopic residues $(15-20 \mathrm{~mm})$, while SPECT only one of these, a mucinous $\mathrm{BC}$, in which $\mathrm{DBC}$ was not performed. DBC correctly classified all other 12 patients in whom the procedure was performed. Both conventional planar SM and SPECT proved useful diagnostic tools in monitoring neoadjuvant chemo/hormono therapy response in LAPBC with SPECT appearing more sensitive; however, our data, although in a limited number of cases, suggest that sensitivity
\end{abstract}

Correspondence to: Dr Giuseppe Madeddu, Department of Nuclear Medicine, University of Sassari, Viale San Pietro 8, I-07100 Sassari, Italy

E-mail: giuseppe.madeddu@email.it

Key words: locally advanced primary breast cancer, neoadjuvant chemotherapy, neoadjuvant hormonotherapy, scintimammography, SPECT, dedicated breast camera, 99mTc-tetrofosmin can further be increased using high resolution DBC, especially in detecting microscopic residual tumor foci.

\section{Introduction}

The initial treatment of choice in patients with locally advanced primary breast cancer (LAPBC) is represented by neoadjuvant therapy (1) to reduce the size of the primary tumor before surgery, thus allowing both a more conservative surgical approach and an improvement of patient survival.

For several years chemotherapy has represented the only available neoadjuvant treatment. However, with recent developments in endocrine therapy, hormonotherapy has been proposed as an alternative approach to chemotherapy in post-menopausal women with large or locally advanced breast tumors with positive estrogen (E) and/or progesterone receptors $(\mathrm{PgR})$, since this treatment has obtained results similar to chemotherapy in overall objective response and breast-conserving surgery, but with the advantage of being better tolerated (2).

The assessment of local tumor response to neoadjuvant treatment is extremely important in planning the most appropriate surgical treatment as well as in selecting post-operative therapies; this is generally based on the employment of conventional diagnostic methods, such as clinical and mammographic/ultrasonographic procedures which, however, have proved of limited value, over or underestimating residual disease extension $(3,4)$, due to the difficulties these methods demonstrate in differentiating residual tumors from fibrosis replacement.

Conventional planar scintimammography (SM) with cationic lipophilic gamma-emitting radiotracers acquired with a general purpose gamma camera have proved more suitable than the aforementioned conventional imaging methods in monitoring the response to neoadjuvant chemotherapy (5), especially in excluding post-treatment residual tumors, although false negative results can occur at SM, in particular when the lesions are small in size $(5,6)$.

Preliminary studies have demonstrated that single photon emission computed tomography (SPECT) with cationic lipophilic radiotracers can be useful for monitoring neoadjuvant chemotherapy (7). This procedure, giving high 
contrast and cross-sectional images, has proved to increase the sensitivity of conventional planar SM in primary breast carcinoma detection, especially when the lesions are nonpalpable and of small size $(8,9)$.

However, at present, no comparative study between conventional planar SM and SPECT exists in monitoring neoadjuvant chemotherapy, nor have either of these two procedures been used in the evaluation of the response to the recently developed neoadjuvant hormonotherapy.

The aim of the present study was to comparatively evaluate the usefulness of conventional planar SM and SPECT in monitoring the response to neoadjuvant chemo- or hormonotherapy in patients with LAPBC, using the cationic lipophilic $99 \mathrm{mTc}$-tetrofosmin as radiotracer. As well as the 2 above diagnostic procedures, a further SM was acquired in our more recent cases using a newly developed high resolution dedicated breast camera (DBC) which has recently proved a highly sensitive tool in early primary breast cancer identification $(10,11)$.

\section{Materials and methods}

Patients. We studied 32 consecutive female patients, aged 26-83 years (median age: 52 years), with LAPBC, scheduled to undergo neoadjuvant therapy before surgery. In all patients both an accurate history and clinical examination were undertaken by the oncologist of reference and followed by conventional diagnostic imaging procedures (mammography/ ultrasonography) as well as tissue biopsy by fine-needle aspiration biopsy or incisional biopsy. Measurements of tumor histologic grade and in vitro assessment of ER and Pg-R expression, c-erb-B2 (HER-2/neu) gene expression and Ki-67 (MIB1) index of tumor proliferation were performed on biopsy samples.

At biopsy, an infiltrating ductal carcinoma was ascertained in 24 cases, an invasive tumor not otherwise specified in 4 cases, a mucinous carcinoma in 2 cases, a lobular carcinoma in one case and a tubular carcinoma in the remaining case.

On the basis of the results of these diagnostic assessments, 22/32 patients (median age: 47.5 years, range: 26-60 years) were considered eligible for neoadjuvant chemotherapy with a weekly cisplatin-based therapy (cisplatin $30 \mathrm{mg} / \mathrm{m}^{2}$, epirubicin $50 \mathrm{mg} / \mathrm{m}^{2}$ and paclitaxel $120 \mathrm{mg} / \mathrm{m}^{2}$ on day $1 ; 8$ weekly cycles), while the remaining 10 patients, all elderly (median age: 80 years, range: $72-83$ years) and with ERpositive breast tumors, were considered eligible for hormonal endocrine treatment with aromatase inhibitors (exemestane $25 \mathrm{mg}$ /day for 4 months).

The pre-therapy clinico-pathological characteristics of the 32 of patients scheduled for either chemo or hormonotherapy are reported in Table I. The clinical staging was established following the American Joint Committee on Cancer (AJCC) criteria (12). At the end of the therapeutic cycles, all patients were rechecked by both clinical and conventional diagnostic imaging procedures and surgery was planned for 2 weeks later.

Patients with concomitant distant metastases ascertained at standard staging diagnostic procedures (chest X-ray, bone scan and abdomen US) and patients scheduled for concomitant radiotherapy were excluded from this study.
Table I. Pre-therapy clinico-pathological characteristics of the 32 patients with locally advanced primary breast cancer (LAPBC) scheduled for either chemo- or hormonotherapy.

\begin{tabular}{ccc}
\hline & $\begin{array}{c}\text { Patients submitted } \\
\text { to chemotherapy } \\
(n=22)\end{array}$ & $\begin{array}{c}\text { Patients submitted } \\
\text { to hormonotherapy } \\
(n=10)\end{array}$ \\
\hline
\end{tabular}

Age at diagnosis

(years)

$<50$

11

0

$\geq 50$

11

10

Clinical tumor stage

$\mathrm{T} 2$

$6 \quad 9$

T3

7

0

T4

9

1

Clinical lymph

NO

N1

12

$\mathrm{N} 2$

Pre-menopausal

status

Post-menopausal

status

ER-positive tumors

ER-negative tumors

8

0

Scintimammography. All 32 patients underwent both conventional planar SM and SPECT using 99mTc-tetrofosmin (Myoview, GE Healthcare) as radiotracer twice: at the moment of the clinical diagnosis and again after chemo or hormonotherapy cycles, 2-3 days before surgery. Together with conventional planar SM and SPECT, the last 15 patients enrolled in the study also underwent DBC planar SM, 4 of whom only once at the control after therapy, and 11 before and after treatment.

The protocol of the present study was in accordance with the Helsinki Doctrine on Human Experimentation and written informed patient consent was always obtained before scintigraphy. Radiolabelling and quality control procedures of the radiotracer were carried out according to the manufacturer's instructions. Labelling efficiency was always over $95 \%$. In all patients, $740 \mathrm{MBq}$ of $99 \mathrm{mTc}$-tetrofosmin were intravenously injected into the arm contralateral to the mammary lesion. Ten minutes later, conventional planar SM was performed, using a rectangular, large field of view, dual-head gamma camera (Helix, Elscint or Millennium VG, GE Healthcare) equipped with low-energy, high-resolution, parallel-hole collimators. A $\pm 10 \%$ energy window and a $140 \mathrm{KeV}$ photo-peak were selected. Planar 
images were acquired both in anterior and in lateral views, with a 256x256 matrix size, an acquisition time of $600 \mathrm{sec}$ per view and a zoom factor ranging from 1-1.2, according to the individual patient.

After planar scintigraphy, SPECT images were acquired over $360^{\circ}\left(180^{\circ}\right.$ per head) in supine position with the same zoom factor as used for the anterior planar view, with a $64 \times 64$ matrix size, a $3^{\circ}$ angular step and an acquisition time of $30 \mathrm{sec} /$ frame. The body contouring system was always used in order to ensure the minimum distance between the patient and the collimators. SPECT image reconstruction was performed with the Back Projection Filter Method with a Metz filter (coefficient: 3; FWHM: 10). Transaxial slices were used to obtain coronal and sagittal slices.

In the aforementioned 15 patients, following both conventional planar and SPECT, high resolution planar SM was also acquired using a LumaGEM 3200S/12k (Gamma Medica Ideas Inc.), high-resolution, solid-state dedicated breast camera, which represents the latest advance in LumaGEM breast cameras. The detector is attached to an adaptor mounted on a modified mammographic unit, replacing the radiographic Bucky, permitting projections very similar to those of mammography. The camera field of view is $20 \times 15 \mathrm{~cm}$. The camera head is composed of a semiconductor detector with a pixelated $(12,288$ pixels; pixel size: $1.5 \times 1.5 \times 5 \mathrm{~mm}$ ) array of cadmium-zinc-thelluride (CZT) coupled to an array of amplifiers, the signals from which are conveyed on an electronic readout board. The system is equipped with a highly sensitive (HSEN, LEAP) long-bore, low-energy collimator (hole shape: hex; hole length: $25.4 \mathrm{~mm}$; hole diameter: $2 \mathrm{~mm}$; septal thick: $0.3 \mathrm{~mm}$ ) matched to the CZT elements. The system is modeled to have an intrinsic spatial resolution of $1.6 \mathrm{~mm}$ and an energy resolution $<5 \%$ (average $4.6 \%$ at $140 \mathrm{Kev}$ ). In all cases the breast images were acquired both in craniocaudal and mediolateral oblique projections (600 sec per view), using a $128 \times 128$ matrix size, with the breast positioned between the detector and the compression paddle of the mammographic unit to ensure a light compression of the breast parenchyma, reducing its thickness, limiting movement artefacts and improving lesion contrast. Additional projections could be acquired when necessary (such as with breast bigger than the field of view, areas of increased uptake at the border of the field of view, etc.), given the flexibility of mammographic gantry in patient positioning.

Planar and SPECT images were independently evaluated by visual analysis by two experienced nuclear medicine physicians (A.S. and G.M.) who were blinded to the clinical findings and to the other diagnostic imaging procedure data. Planar SM, both by conventional and DBC camera method acquisition results, were compared to those of SPECT, and both of these were subsequently related to the histopathological findings obtained after surgery.

Histopathological diagnosis. Pathological analysis was performed in all patients on surgical specimens. A macroscopic analysis was performed with the size of residual tumors determined according to the largest diameter. Breast surgical specimens were then fixed in $10 \%$ buffered formalin and stained with haematoxylin and eosin. A further immuno- histochemical analysis was performed with microscopic evaluation.

Response evaluation. Clinical and mammographic assessments were performed measuring the largest tumor diameter: complete remission (CR) was defined as total disappearance of primary tumor; no remission (NR) in the presence of slight or no reduction in tumor size; partial remission (PR) in the presence of significant $(>50 \%)$ reduction in tumor size. Scintigraphic images were considered as indicative of $\mathrm{CR}$ in the absence of detectable uptake on the post-therapy scan, indicative of NR in the presence of faint or no reduction in tumor uptake and indicative of PR in the presence of significant reduction $(>50 \%)$ in tumor uptake by visual analysis.

The pathological response to chemo/hormone neoadjuvant therapy was classified as follows: pathological complete remission ( $\mathrm{pCR}$ ) with no evidence of residual tumor at both macroscopic and microscopic pathological evaluation; near total pathological complete remission (NTpCR) with evidence of residual tumor only at microscopic pathological evaluation; pathological partial remission (pPR) with residual tumor at histopathological analysis $<50 \%$ in its largest diameter compared with initial data obtained at mammography evaluation; pathological no remission $(\mathrm{pNR})$ with residual tumor at histopathological analysis $>50 \%$ in its largest diameter compared with initial data or even with progressive disease.

Statistical analysis. 99mTc-tetrofosmin planar and SPECT images were classified as true-positive, true-negative, falsepositive or false-negative considering the histopathological findings obtained at surgery as the 'gold standard'. Sensitivity (i.e. true positive results or percentage of correct prediction of residual tumor presence after neoadjuvant therapy) and specificity (i.e. true-negative results or percentage of correct prediction of residual tumor absence after neoadjuvant therapy) were then calculated on this basis. McNemar's test was used to assess the statistical differences in sensitivity and specificity between conventional planar SM and SPECT. The results were considered significant when P-value was $<0.05$. The results obtained with DBC planar SM were excluded from statistical evaluations, due to the small number of cases studied with this procedure.

\section{Results}

At the first observation, before starting neoadjuvant chemo or hormonotherapy, all 32 LAPBC patients were true-positive for primary breast carcinoma at both $99 \mathrm{mTc}$-tetrofosmin conventional planar SM and SPECT; DBC planar SM was also true positive in the 11 patients also submitted to this procedure.

The individual pathological response data ascertained at surgery in all patients in relation to the scintigraphic results are reported in Table II, which also illustrates pre-therapy TNM clinical staging and histological type as well as the kind of treatment. Four of 32 patients, 3 after chemo- and one after hormonotherapy, had pCR at surgery; 2/32 patients, both after chemotherapy, had NTpCR with multiple sub- 
Table II. Individual patient data on pathological and scintigraphic response to neoadjuvant chemo- or hormonotherapy in 32 patients with locally advanced primary breast cancer (LAPBC).

\begin{tabular}{|c|c|c|c|c|c|c|c|c|c|c|}
\hline No. & Age & $\begin{array}{l}\text { Histologic } \\
\text { type }\end{array}$ & $\begin{array}{c}\text { Tumor } \\
\text { size }(\mathrm{cm}) \\
\text { before } \\
\text { therapy } \\
\text { at } \mathrm{Mx}\end{array}$ & $\begin{array}{l}\text { Clinical } \\
\text { staging } \\
\text { (TNM) }\end{array}$ & $\begin{array}{c}\text { Treatment } \\
\text { type }\end{array}$ & $\begin{array}{c}\text { Pathological } \\
\text { response }\end{array}$ & $\begin{array}{c}\text { Tumor } \\
\text { size }(\mathrm{cm}) \\
\text { at } \\
\text { histology }\end{array}$ & $\begin{array}{l}\text { Planar } \\
\text { SM } \\
\text { response }\end{array}$ & $\begin{array}{c}\text { SPECT } \\
\text { response }\end{array}$ & $\begin{array}{c}\text { DBC } \\
\text { planar } \\
\text { SM } \\
\text { response }\end{array}$ \\
\hline 1 & 59 & IDCa & 4.5 & T2N1 & Chemo & pNR & 4.5 & NR & NR & NP \\
\hline 2 & 31 & IDCa & 6 & T3N2 & Chemo & $\mathrm{pNR}$ & 4 & NR & NR & NP \\
\hline 3 & 50 & IDCa & 4.5 & T2N1 & Chemo & pNR & 3.5 & NR & NR & NP \\
\hline 4 & 29 & IDCa & 6 & T3N2 & Chemo & pNR & 6 & NR & NR & NP \\
\hline 5 & 83 & IDCa & 5 & T2NO & Hormono & pNR & 4.5 & NR & NR & NP \\
\hline 6 & 41 & IDCa & 10 & T4N1 & Chemo & $\mathrm{pCR}$ & 0 & $\mathrm{CR}$ & $\mathrm{CR}$ & NP \\
\hline 7 & 47 & IDCa & 5 & T4N1 & Chemo & $\mathrm{pCR}$ & 0 & $\mathrm{CR}$ & CR & NP \\
\hline 8 & 45 & IDCa & 3.5 & T4N1 & Chemo & $\mathrm{pNR}$ & 2.2 & NR & NR & NP \\
\hline 9 & 52 & NOS & 4 & T4N1 & Chemo & pNR & 3 & NR & NR & NP \\
\hline 10 & 32 & NOS & 5 & T4N1 & Chemo & pPR & 2.5 & PR & PR & NP \\
\hline 11 & 72 & IDCa & 6 & T4N2 & Hormono & pPR & 3 & PR & PR & NP \\
\hline 12 & 52 & IDCa & 5 & T4N1 & Chemo & pPR & 2 & PR & PR & NP \\
\hline 13 & 45 & IDCa & 5.5 & T3N2 & Chemo & pPR & 2.5 & PR & PR & NP \\
\hline 14 & 81 & IDCa & 3.5 & T2NO & Hormono & pPR & 1.5 & $\mathrm{CR}$ & PR & NP \\
\hline 15 & 81 & IDCa & 3 & $\mathrm{~T} 2 \mathrm{~N} 0$ & Hormono & pNR & 2 & NR & NR & NP \\
\hline 16 & 82 & IDCa & 3.5 & T4NO & Hormono & pCR & 0 & CR & CR & NP \\
\hline 17 & 60 & $\mathrm{MuCa}$ & 4 & T2N1 & Chemo & pPR & 1.8 & $\mathrm{CR}$ & $\mathrm{CR}$ & NP \\
\hline 18 & 50 & IDCa & 4 & T2NO & Chemo & pPR & 1.2 & PR & PR & PR \\
\hline 19 & 82 & IDCa & 5 & T2NO & Hormono & $\mathrm{pPR}$ & 2 & $\mathrm{CR}$ & PR & PR \\
\hline 20 & 77 & IDCa & 5 & T3NO & Hormono & pPR & 1.5 & PR & PR & PR \\
\hline 21 & 36 & IDCa & 3.5 & T2NO & Chemo & $\mathrm{pCR}$ & 0 & $\mathrm{CR}$ & $\mathrm{CR}$ & CR \\
\hline 22 & 26 & IDCa & 3 & T2NO & Chemo & NTpCR & $<1$ & $\mathrm{CR}$ & $\mathrm{CR}$ & PR \\
\hline 23 & 79 & ILCa & 3 & $\mathrm{~T} 2 \mathrm{~N} 1$ & Hormono & pPR & 1 & PR & PR & PR \\
\hline 24 & 48 & IDCa & 5 & T4N2 & Chemo & pPR & 1.5 & PR & PR & PR \\
\hline 25 & 55 & IDCa & 3.5 & $\mathrm{~T} 2 \mathrm{~N} 1$ & Chemo & pPR & 1.5 & PR & PR & PR \\
\hline 26 & 33 & IDCa & 5 & T3N1 & Chemo & pPR & 2 & PR & PR & PR \\
\hline 27 & 40 & $\mathrm{TuCa}$ & 4.4 & T3N1 & Chemo & $\mathrm{pNR}$ & 3 & NR & NR & NR \\
\hline 28 & 52 & $\mathrm{MuCa}$ & 5 & T3N2 & Chemo & pNR & 7 & NR & NR & NR \\
\hline 29 & 57 & IDCa & 5.5 & T2NO & Chemo & $\mathrm{pPR}$ & 2.5 & PR & PR & PR \\
\hline 30 & 59 & NOS & 5 & T4N1 & Chemo & NTpCR & $<1$ & $\mathrm{CR}$ & $\mathrm{CR}$ & PR \\
\hline 31 & 79 & NOS & 4.5 & T2N1 & Hormono & $\mathrm{pPR}$ & 2 & PR & PR & PR \\
\hline 32 & 72 & IDCa & 4 & $\mathrm{~T} 2 \mathrm{~N} 2$ & Hormono & pNR & 5.5 & NR & NR & NR \\
\hline
\end{tabular}

No., patient number; IDCa, infiltrating ductal carcinoma; NOS, invasive tumor not otherwise specified; ILCa, infiltrating lobular carcinoma; $\mathrm{TuCa}$, tubular carcinoma; $\mathrm{MuCa}$, mucinous carcinoma; $\mathrm{Mx}$, mammography; pNR, pathological no remission; pCR, pathological complete remission; pPR, pathological partial remission; NTpCR, near total pathological complete remission; NR, CR and PR, no remission, complete remission and partial remission at planar SM, SPECT and DBC; NP, not performed.

centimetric tumor foci scattered in a fibrotic area, invasive in one case and in situ in the other; $15 / 32$ patients, 9 after chemo- and 6 after hormonotherapy, had PR with residual tumors ranging in size from 10-25 mm (average size: 18.3 $\mathrm{mm}$ ); the remaining $11 / 32$ patients, 8 after chemo- and 3 after hormonotherapy, had NR ( 9 cases), or even progressive disease ( 2 cases), with residual tumors ranging from 20-70 $\mathrm{mm}$ (average size: $38.4 \mathrm{~mm}$ ).

Considering the response to treatment on the basis of the two different types of therapy, $22.7 \%$ of patients submitted to chemotherapy and $10 \%$ of those submitted to hormonotherapy had CR or NTpCR, while $40.9 \%$ of the former and 

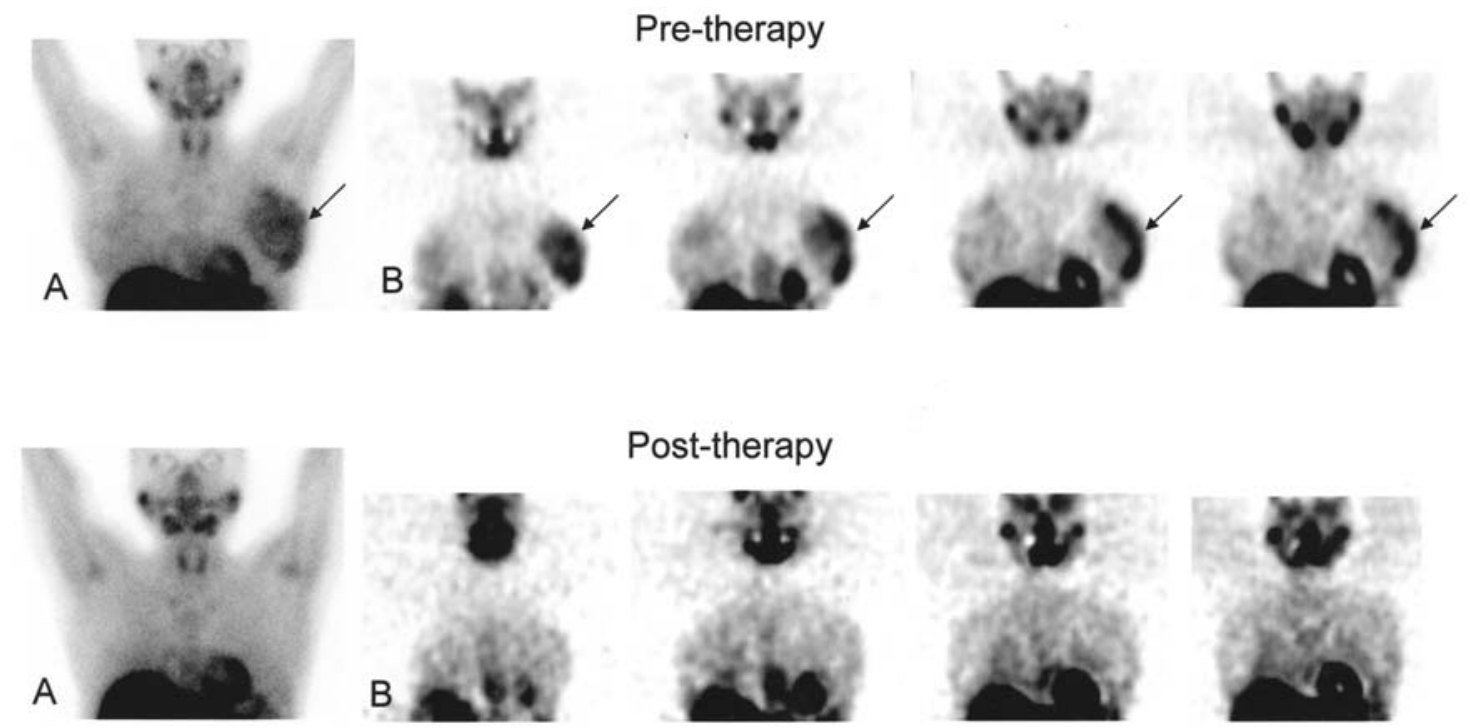

\section{Post-therapy}
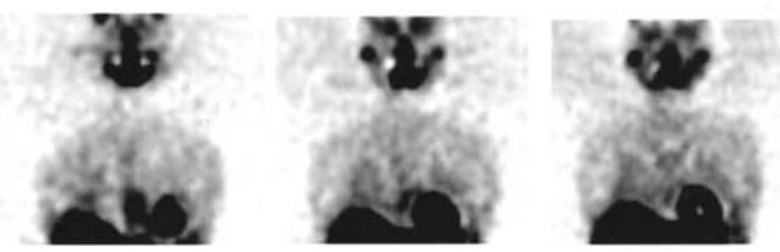

Figure 1. A patient (case no. 6) with a locally advanced primary breast cancer in the left breast, evidenced in the pre-therapy phase at both conventional planar $\mathrm{SM}$ in anterior view (A) and SPECT in multiple coronal slices (B). A complete remission after neoadjuvant chemotherapy was observed at both procedures in accordance with pathological findings.

\section{Pre-therapy}
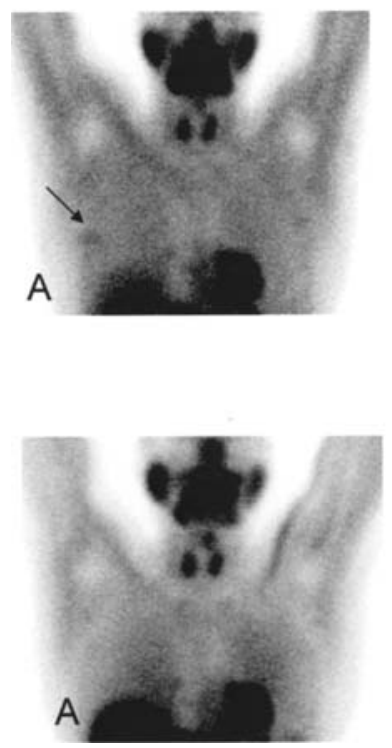

Pre-therapy

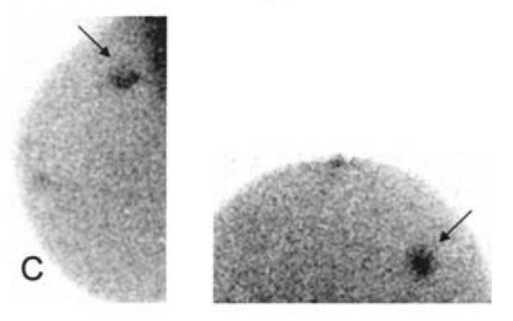

Post-therapy
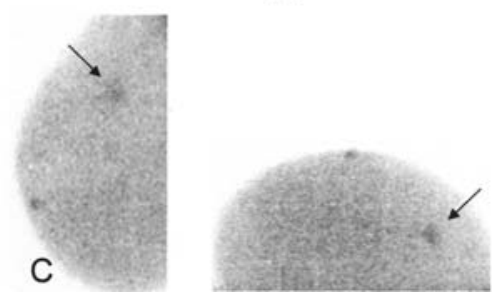

\section{Post-therapy}
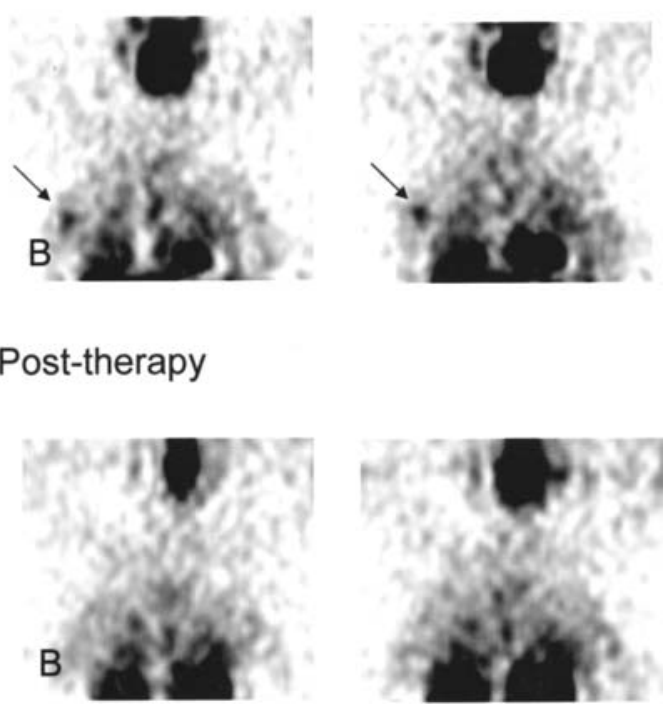

Figure 2. A patient (case no 22) with a locally advanced primary breast cancer in the external upper quadrant of the right breast evidenced in the pre-therapy phase at conventional planar SM (A), SPECT (B) and DBC planar SM (C). A near total pathological complete remission after neoadjuvant chemotherapy was observed, with the multifocal microscopic scattered residual tumors evident only at DBC planar SM while missed at both conventional planar SM and SPECT.

$60 \%$ of the latter had PR; moreover, $36.4 \%$ of patients treated with chemotherapy and $30 \%$ of those treated with hormonotherapy had NR.

Both conventional planar SM and SPECT ascertained $\mathrm{CR}$ with absence of tracer uptake in the post-therapy study in all 4 patients with pCR (one of these cases is illustrated in Fig. 1), as did DBC planar SM in the only responder patient in whom the procedure was performed. Clinical examination correctly predicted pCR in only 2 of these 4 cases, while mammography in only 1 of these 2 , globally missing $3 / 4$. 


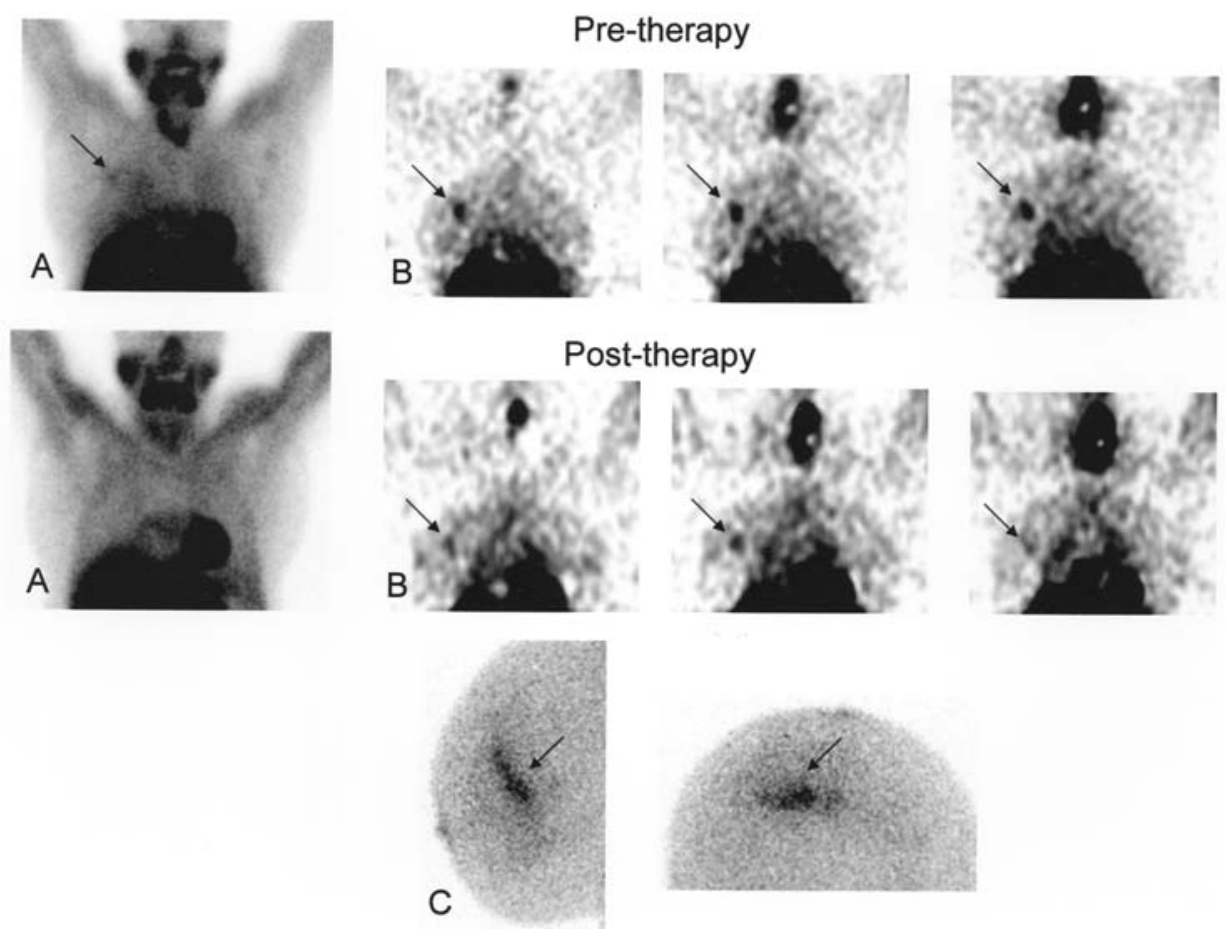

Figure 3. A patient (case no. 19) with a locally advanced primary breast cancer in the internal upper quadrant of the right breast, evidenced in the pre-therapy phase at both conventional planar SM in anterior view (A) and SPECT in multiple coronal slices (B). Conventional planar SM missed the residual tumor, while SPECT was concordant with pathological findings, showing a persistent, but reduced, focal uptake in the post-hormonotherapy study. The residual tumor was also seen at DBC planar SM (C), in cranio-caudal and medio-lateral oblique views, which, however, was only performed after therapy.

Both conventional planar SM and SPECT failed the identification of multifocal microscopic scattered residual tumors in the 2 patients with NTpCR; a persistent tracer uptake, although markedly reduced in respect of the basal study, was evident only at DBC planar SM in both cases (Fig. 2), suggesting the presence of residual disease. Residual tumor size was overestimated in both these cases at clinical examination, while mammography identified only one small focal lesion, failing the diagnosis of multifocality, in 1 case, and was inconclusive in assessing tumor response in the other case because of highly radio-opaque breast tissue.

In the 15 patients with pPR, conventional planar SM and SPECT showed a persistent, but reduced, focal uptake in $12 / 15$ and in 14/15 cases, respectively, as did DBC planar $\mathrm{SM}$ in the 5 cases in this group in whom the procedure was performed before and after treatment. Tracer uptake was also evident at DBC planar SM in 4 other patients in this group in whom the procedure was performed only after therapy. Tracer uptake was not evident at the conventional planar SM performed after treatment in $3 / 15$ patients (one of these cases is shown in Fig. 3) who had macroscopic residual tumors at surgery ranging in size from 15-20 mm, including a mucinous carcinoma, the only macroscopic residue missed by SPECT. The size of this residual mucinous tumor was, however, overestimated at both clinical examination and mammography, and thus incorrectly classified, persisting nipple retraction and microcalcifications. Among the 14 residual tumors detected at SPECT, 4 were overestimated in size at both clinical examination and mammography; 2 further cases were clinically overestimated and 2 other cases were inconclusive at mammography due to high radio-opaque
Table III. Comparative conventional planar SM and SPECT results after chemo- or hormonotherapy in 32 patients with locally advanced primary breast cancer (LAPBC).

\begin{tabular}{lcc}
\hline & $\begin{array}{c}\text { Conventional } \\
\text { planar SM }\end{array}$ & SPECT \\
\hline True negative & 4 & 4 \\
False positive & 0 & 0 \\
True positive & 23 & 25 \\
False negative & 5 & 3 \\
Sensitivity & $82 \%$ & $89.2 \%$ \\
Specificity & $100 \%$ & $100 \%$ \\
Accuracy & $84.4 \%$ & $90.6 \%$
\end{tabular}

Mc Nemar' test for the statistical analysis.

breast tissue. No significant reduction or even increased tracer uptake were observed at either conventional planar SM or SPECT in the remaining $11 \mathrm{pNR}$ patients, as also resulted at $\mathrm{DBC}$ planar $\mathrm{SM}$ in the 3 patients in this group in whom the procedure was performed. Both clinical examination and mammography were concordant with scintigraphic data in assessing no response or progressive disease in all these 11 cases. When conventional planar SM and SPECT were mutually compared, SPECT showed higher sensitivity and accuracy values, although not significantly, as shown in Table III. 


\section{Discussion}

Up to date chemotherapy has represented the neoadjuvant treatment of choice in LAPBC patients. More recently, hormonotherapy, a relatively new treatment option, has also been proposed in elderly and post-menopausal women with ER-positive breast cancer (13). In particular, a growing interest has recently been registered in the third generation aromatase inhibitors $(14,15)$, including exemestane, which act by blocking the estrogen synthesis and have proven more efficacious than the standard estrogen receptor modulator drugs, also presenting the advantage of having no intrinsic estrogenic activity. Moreover, phase II randomized trials have demonstrated the superiority of preoperative neoadjuvant endocrine therapy with the aromatase inhibitor exemestane in respect of chemotherapy in both overall objective response leading to breast-conserving surgery in post-menopausal women (2). A wider employment of neoadjuvant endocrine therapy is thus expected in the future in selected breast cancer patients, also because it is better tolerated in respect of standard chemotherapy.

In the present study, performed in LAPBC patients before and after chemo- or hormone adjuvant therapy, a pCR following treatment was observed in $12.5 \%$ of cases, while residual tumors were found at surgery in the remaining $87.5 \%$ of cases. However, in $7 \%$ of the latter non-responder patients, a NTpCR was obtained, the residual tumors being very tiny and scattered in fibrotic area and visible only at microscopic pathological evaluation. Chemotherapy and hormonotherapy were associated with similar results, globally obtaining complete or partial remission in 63.6 and $70 \%$ of cases, respectively.

The data obtained in our series suggest that radioisotopic imaging procedures are valuable diagnostic tools for monitoring the efficacy of neoadjuvant treatments in local disease control since $99 \mathrm{mTc}$-tetrofosmin SM, both conventional planar and SPECT, excluded the presence of residual tumor in $100 \%$ of cases with pCR following either chemo- or hormonotherapy, demonstrating a better performance in respect of both clinical examination and mammography, which were concordant with pathological data in 50 and $25 \%$ of cases, respectively.

On the other hand, it is known that both clinical examination and conventional diagnostic imaging tools, such as mammography and ultrasound, are poorly efficacious in ascertaining complete pathological remission $(5,16)$, due to the difficulties of these procedures in differentiating residual tumors from tissue challenges, such as fibrosis, induced by the neoadjuvant drugs.

As demonstrated in in vitro studies, tetrofosmin uptake in tumoral cells is favoured by both increased blood flow and capillary permeability and also by elevated metabolic activity (17), and is strictly dependent on cell membrane and mitochondria potentials $(18,19)$ and partially related to $\mathrm{Na}^{+} / \mathrm{K}^{+}$pump and $\mathrm{Na}^{+} / \mathrm{H}^{+}$antiport systems (20); consequently, only viable cells, but not fibrotic tissues, can show radiotracer uptake. These uptake mechanisms explain the absence of false positive findings in our series and, in general, the better intrinsic specificity of a functional imaging, such as $\mathrm{SM}$, in respect of conventional diagnostic non radioiso- topic imaging procedures purely based on anatomo-structural lesion volume changes.

The above is confirmed also in other studies focussing on patients submitted to neoadjuvant chemotherapy in which SM did not show false positive findings, with a higher specificity than clinical examination and mammography (5). In these studies, the cationic lipophilic $99 \mathrm{mTc}$-sestaMIBI was used as radiotracer, although this presents biochemical properties and tumoral uptake mechanisms very similar to those of tetrofosmin (17-20).

The patient by patient comparative evaluation performed in the present study demonstrated that similarly high values of specificity exist with conventional planar SM and SPECT, but SPECT proved more sensitive in assessing the presence of residual tumors, although the difference was not statistically significant.

The higher sensitivity of SPECT in tumor detection in respect of conventional planar SM has already been demonstrated in early primary breast carcinoma detection $(8,9)$, in which, however, the performance of both procedures appears to be prevalently dependent on tumor size, although this factor seems to affect planar SM more than SPECT (21-23). In our series, SPECT identified two macroscopic residual carcinomas sized 15 and $18 \mathrm{~mm}$ but missed at conventional planar SM. However, in 2 NTpCR patients neither planar SM nor SPECT managed to detect two further minimal residual tumors, one in situ, both characterized by multiple subcentimetric tumor foci scattered in a fibrotic area, with a size below the intrinsic spatial resolution of the conventional gamma camera used for image acquisition. Moreover, both procedures failed the identification of another macroscopic residual tumor, related to a mucinous histotype, which is known to be generally characterized by low cellularity and slow growth rate; this suggests that not only size but also biological factors can affect tumor detection in scintigraphy.

The physical limitations of planar SM, and in part also those of SPECT, in detecting small tumoral lesions unfortunately are not surmountable with conventional devices.

These considerations explain the recent growing interest in the development of DBC, a new compact device specifically designed for imaging breasts, which is characterized by a markedly higher spatial resolution in respect of conventional general purpose gamma cameras and at the same time offers several technical advantages in breast image acquisition, such as the exclusion of nearby organs from the field of view and reduced distance between lesions and detector; in addition, since the detector can be inserted into a mammograph, it is possible to obtain similar projections to the mammography as well as mild breast compression during acquisition.

Preliminary comparative studies have demonstrated that planar SM acquired with DBC gives a significantly higher sensitivity than conventional planar SM in non-palpable and early subcentimetric primary carcinomas $(24,25)$; DBC has also proved able to further increase the sensitivity of SPECT, although not significantly (11).

The data of the present study demonstrated that DBC planar SM could also play an important role in monitoring the response to neoadjuvant chemo- and hormonotherapy 
in LAPBC, increasing the sensitivity of conventional radioisotopic methods in small size residual tumor detection. In particular, in our series, DBC planar SM proved the only radioisotopic procedure able to achieve the goal of identifying minimal residual disease in 2 patients, as mentioned above; neither clinical examination nor mammography proved accurate in the assessment of tumor response in either of these cases, the former method overestimating the degree of residual disease in both, while the latter failed the diagnosis of multifocality in one case and was indeterminate for highly dense radio-opaque breast tissue in the other one. These results, although in a small number of cases, would seem to indicate that DBC planar SM has the potential of improving the sensitivity of conventional planar SM and SPECT as well as overcoming the main drawbacks of conventional diagnostic non radioisotopic imaging procedures. This high performance of DBC appears even more important given the many limitations of the latter aforementioned procedures, such as scattered tumor cells in a fibrotic area, remaining intraductal carcinoma after the disappearance of the invasive component, radio-opaque dense breast, remaining microcalcifications and lobular histotype, which represent the more frequent features that may not be accurately characterized by mammography (26-28). Scattered tumor cells and intraductal carcinomas have proved difficult to assess even by the more sophisticated dynamic contrast-enhanced magnetic resonance imaging (DCE-MRI), which has been proposed by some authors $(29,30)$ as an alternative to standard anatomic procedures, such as mammography and ultrasound, in assessing tumor response to neoadjuvant chemotherapy. The usefulness of DCE-MRI in this field is, however, still debated, with conflicting results reported in literature, also in relation to the different analysis methods used for dynamic image interpretation $(29,31,32)$.

A wider employment of DBC planar SM is thus suggested, since it could contribute to a more accurate classification of patients as responder or non-responder following neoadjuvant chemo or hormonotherapy, as observed in our cases, in whom DBC correctly classified all patients who underwent this procedure, although the casuistry was limited.

DBC proved very simple to perform, is well tolerated and the images show an extremely high resolution and contrast and are prompt and clear to read; moreover, the images are also easily comparable with the corresponding projections of mammography, giving a precise localization of residual tumors within the breast.

Thus, DBC planar SM could also represent a useful guide for the surgeon in planning the most appropriate surgical treatment, selecting the patients for conservative breast surgery with the intent of achieving even negative margins and optimal cosmesis. However, up to date, DBCs are still poorly available and under development for optimizing collimation and electronic systems to further increase their performance and reliability.

Where DBCs are not available, conventional planar SM and SPECT methods can be used, since in our study they demonstrated a better performance than standard non radioisotopic methods, as mammography, especially in assessing complete pathological response and in characterizing the real extension of residual disease due to their ability in differentiating tumor from fibrosis replacement. However, SPECT should be preferred to conventional planar SM, since it showed a higher sensitivity in macroscopic residual tumor detection, although not significantly, in our cases. SPECT images are simple to perform and easy to read, although a more adequate training is necessary in respect of both conventional and DBC planar SM.

Moreover, it should be borne in mind that while the presence of focal areas of uptake, either at conventional planar SM or SPECT, seem to be associated with the presence of residual tumor, the absence of uptake cannot rule out malignancy, especially in cases with either minimal residual disease or tumor histotypes characterized by low metabolism and slow growth rate.

However, we are aware that the data obtained in the present study refer to a small number of cases and that they should be confirmed in a larger cohort. In particular, a wider use of DBC should be suggested in selected patients.

In conclusion, both $99 \mathrm{mTc}$-tetrofosmin conventional planar SM and SPECT proved useful diagnostic tools in monitoring the response to neoadjuvant chemo- or hormonotherapy in LAPBC patients, although SPECT appeared more sensitive than conventional planar SM in detecting residual tumors. However, our data, although in a small number of cases, seem to suggest that the sensitivity of these two procedures further increases by using a very high resolution DBC, especially in microscopic residual tumor foci detection.

\section{References}

1. Booser DJ and Hortobagyi GN: Treatment of locally advanced breast cancer. Semin Oncol 19: 278-285, 1992.

2. Semiglazov VF, Semiglazov VV, Dashyan GA, Zitsova EK, Ivanov VG, Bozhok AA, Melnikova OA, Paltuev RM, Kletzel A and Berstein LM: Phase 2 randomized trial of primary endocrine therapy vs. chemotherapy in post-menopausal patients with estrogen receptor-positive breast cancer. Cancer 110: 244-254, 2007.

3. Sperber F, Weinstein Y, Sarid D, Ben Yosef R, Shalmon A and Yaal-Hahoshen N: Preoperative clinical, mammographic and sonographic assessment of neoadjuvant chemotherapy response in breast cancer. Isr Med Assoc J 8: 342-346, 2006.

4. Chagpar AB, Middleton LP, Sahin AA, Dempsey P, Buzdar AU, Mirza AN, Ames FC, Babiera GV, Feig BW, Hunt KK, Kuerer HM, Meric-Bernstam F, Ross MI and Singletary SE: Accuracy of physical examination, ultrasonography, and mammography in predicting residual pathologic tumor size in patients treated with neoadjuvant chemotherapy. Ann Surg 243: 257-264, 2006.

5. Maini CL, Tofani A, Sciuto R, Semprebene A, Cavaliere R, Mottolese M, Benevolo M, Ferranti F, Grandinetti ML, Vici P, Lopez M and Botti C: Technetium-99m-MIBI scintigraphy in the assessment of neoadjuvant chemotherapy in breast carcinoma. J Nucl Med 38: 1546-1551, 1997.

6. Marshall C, Eremin J, El-Sheemy M, Eremin O and Griffiths PA: Monitoring the response of large $(>3 \mathrm{~cm})$ and locally advanced (T3-4, N0-2) breast cancer to neoadjuvant chemotherapy using (99m)Tc-Sestamibi uptake. Nucl Med Commun 26: 9-15, 2005.

7. Tiling R, Linke R, Untch M, Richter A, Fieber S, Brinkbaumer K, Tatsch K and Hahn K: 18F-FDG PET and 99mTc-sestamibi scintimammography for monitoring breast cancer response to neoadjuvant chemotherapy: a comparative study. Eur J Nucl Med 28: 711-720, 2001.

8. Spanu A, Dettori G, Nuvoli S, Porcu A, Falchi A, Cottu P, Solinas ME, Scanu AM, Chessa F and Madeddu G: 99mTctetrofosmin SPET in both primary breast cancer and axillary lymph node metastasis detection. Eur J Nucl Med 28: 1781-1794, 2001 . 
9. Spanu A, Schillaci O, Meloni GB, Porcu A, Cottu P, Nuvoli S, Falchi A, Chessa F, Solinas ME and Madeddu G: The usefulness of $99 \mathrm{mTc}$-tetrofosmin SPECT scintimammography in the detection of small size primary breast carcinomas. Int $\mathbf{J}$ Oncol 21: 831-840, 2002.

10. Rhodes DJ, O'Connor MK, Phillips SW, Smith RL and Collins DA: Molecular breast imaging: a new technique using technetiumTc scintimammography to detect small tumors of the breast. Mayo Clin Proc 80: 24-30, 2005.

11. Spanu A, Cottu P, Manca A, Chessa F, Sanna D and Madeddu G: Scintimammography with dedicated breast camera in unifocal and multifocal/multicentric primary breast cancer detection: a comparative study with SPECT. Int J Oncol 31: 369-377, 2007.

12. Green FL, Page DL, Fleming ID, Fritz AG, Balch CM, Haller DG, Morrow M (eds). AJCC Cancer Staging Manual. 6th edition. Springer, New York, 2002.

13. Mouridsen HT, Rose C, Brodie AH and Smith IE: Challanges in the endocrine management of breast cancer. Breast 12 (Suppl 2): S2-S19, 2003

14. Goss PE: Emerging role of aromatase inhibitors in the adjuvant setting. Am J Clin Oncol 26: S27-S33, 2003

15. Freedman OC, Verma S and Clemons MJ: Using aromatase inhibitors in the neoadjuvant setting: evolution or revolution? Cancer Treat Rev 31: 1-17, 2005.

16. Schott AF, Roubidoux MA, Helvie MA, Hayes DF, Kleer CG, Newman LA, Pierce LJ, Griffith KA, Murray S, Hunt KA, Paramagul $\mathrm{C}$ and Baker LH: Clinical and radiologic assessments to predict breast cancer pathologic complete response to neoadjuvant chemotherapy. Breast Cancer Res Treat 92: 231-238, 2005.

17. Rodriques M, Chehne F, Kalinowska W, Zielinski C and Sinzinger H: Comparative $99 \mathrm{mTc}-\mathrm{MIBI}$, 99mTc-tetrofosmin and $99 \mathrm{mTc}$-furifosmin in human soft tissue sarcoma cell lines. Eur J Nucl Med 27: 1839-1843, 2000.

18. Arbab AS, Koizumi K, Toyama K and Araki T: Uptake of technetium-99m-tetrofosmin, technetium-99m-MIBI and thallium-201 in tumor cell lines. J Nucl Med 37: 1551-1556, 1996.

19. Bernard BF, Krenning EP, Breeman WA, Ensing G, Benjamins H, Bakker WH, Visser TJ and De Jong M: 99mTc-MIBI, 99mTctetrofosmin and 99mTc-Q12 in vitro and in vivo. Nucl Med Biol 25: 233-240, 1998.

20. Arbab AS, Koizumi K, Toyama K, Arai T and Araki T: Ion transport systems in the uptake of 99Tcm-tetrofosmin, 99TcmMIBI and $201 \mathrm{Tl}$ in a tumour cell line. Nucl Med Commun 18: 235-240, 1997

21. Mekhmandarov S, Sandbank J, Cohen M, Lelcuk S and Lubin E: Technetium-99m-MIBI scintimammography in palpable and non-palpable breast lesions. J Nucl Med 39: 86-91, 1998.
22. Howarth D, Sillar R, Clark D and Lan L: Technetium-99m sestamibi scintimammography: the influence of histopathological characteristics, lesion size and the presence of carcinoma in situ in the detection of breast carcinoma. Eur J Nucl Med 26: $1475-1481,1999$.

23. Khalkhali I, Villanueva-Meyer J, Edell SL, Connolly JL, Schnitt SJ, Baum JK, Houlihan MJ, Jenkins RM and Haber SB: Diagnostic accuracy of $99 \mathrm{mTc}-$ Sestamibi breast imaging: multicenter trial results. J Nucl Med 41: 1973-1979, 2000

24. Scopinaro F, Pani R, De Vincentis G, Soluri A, Pellegrini R and Porfiri LM: High-resolution scintimammography improves the accuracy of technetium-99m methoxyisobutylisonitrile scintimammography: use of a new dedicated gamma camera. Eur J Nucl Med 26: 1279-1288, 1999.

25. Brem RF, Schoonjans JM, Kieper DA, Majewski S, Goodman S and Civelek C: High-resolution scintimammography: a pilot study. J Nucl Med 43: 909-915, 2002.

26. Moskovic EC, Mansi JL, King DM, Murch CR and Smith IE: Mammography in the assessment of response to medical treatment of large primary breast cancer. Clin Radiol 47: 339-344, 1993.

27. Vinnicombe SJ, MacVicar AD, Guy RL, Sloane JP, Powels TJ, Knee G and Husband JE: Primary breast cancer: mammographic changes after neoadjuvant chemotherapy, with pathologic correlation. Radiology 198: 333-340, 1996.

28. Peintinger F, Kuerer HM, Anderson K, Boughey JC, Meric-Bernstam F, Singletary SE, Hunt KK, Whitman GJ, Stephens T, Buzdar AU, Green MC and Symmans WF: Accuracy of the combination of mammography and sonography in predicting tumor response in breast cancer patients after neoadjuvant chemotherapy. Ann Surg Oncol 13: 1443-1449, 2006.

29. Pickles MD, Lowry M, Manton DJ, Gibbs P and Turnbull LW: Role of dynamic contrast enhanced MRI in monitoring early response of locally advanced breast cancer to neoadjuvant chemotherapy. Breast Cancer Res Treat 91: 1-10, 2005.

30. Londero V, Bazzocchi M, Del Frate C, Puglisi F, Di Loreto C, Francescutti $\mathrm{G}$ and Zuiani C: Locally advanced breast cancer: comparison of mammography, sonography and MR imaging in evaluation of residual disease in women receiving neoadjuvant chemotherapy. Eur Radiol 14: 1371-1379, 2004.

31. Denis F, Desbiez-Bourcier AV, Chapiron C, Arbion F, Body G and Brunereau L: Contrast enhanced magnetic resonance imaging underestimates residual disease following neoadjuvant docetaxel based chemotherapy for breast cancer. Eur J Surg Oncol 30: 1069-1076, 2004.

32. Kwong MS, Chung GG, Horvath LJ, Ward BA, Hsu AD, Carter D, Tavassoli F, Haffty B and Burtness BA: Postchemotherapy MRI overestimates residual disease compared with histopathology in responders to neoadjuvant therapy for locally advanced breast cancer. Cancer J 12: 212-221, 2006. 